\title{
JUNGLE: An Interactive Visual Platform for Collaborative Creation and Consumption of Nonlinear Transmedia Stories
}

\author{
Mubasir Kapadia ${ }^{1}$, Carlos Manuel Muniz ${ }^{1}$, Samuel S. Sohn ${ }^{1}$, Ye Pan ${ }^{2}$, Sasha Schriber ${ }^{2}$, \\ Kenny Mitchell ${ }^{2,3}$, and Markus Gross ${ }^{2,4}$ \\ 1 Rutgers University, ${ }^{2}$ Disney Research, ${ }^{3}$ Edinburgh Napier University, ${ }^{4}$ ETH Zurich
}

\begin{abstract}
JUNGLE is an interactive, visual platform for the collaborative manipulation and consumption of nonlinear transmedia stories. Intuitive visual interfaces encourage JUNGLE users to explore vast libraries of story worlds, expand existing stories, or conceive of entirely original story worlds. JUNGLE stories utilize multiple media forms including videos, images, and text, and accommodate branching narrative outcomes. We extensively evaluate Jungle using a focused small-scale study and free-form large-scale study with careful protection of study participant privacy. In the small-scale study, users found JUNGLE's features to be versatile, engaging, and intuitive for discovering new content. In the large-scale study, 354 subjects tested JUNGLE in a realistic 45-day scenario. We find that users collaborated on story worlds incorporating various forms of media in multiple (on average two) possible story paths. In particular, we find through initial observations that JUNGLE can evoke creativity: traditionally passive consumers gradually transition into active content creators. Supplementary videos showcasing the JUNGLE system and hypothetical example stories authored using JUNGLE independently hosted here and here.
\end{abstract}

Keywords: storytelling $\cdot$ story authoring $\cdot$ nonlinear transmedia stories.

\section{Introduction}

As the patterns of consuming and creating story content evolve, stories are increasingly generated by many authors working together to create rich, immersive, often interactive, and engaging experiences that are told across multiple media formats. Traditionally passive consumers are now dynamic prosumers, who like to be actively engaged in influencing the outcome of narratives. Existing online platforms and communication systems provide isolated support for collaboration, nonlinearity, or transmedial stories. However, there exists no accessible platform for collaborative authoring and consumption of nonlinear transmedia stories.

There are four key requirements towards meeting this goal. (1) Exploration: It should be intuitive to explore vast libraries of complex, nonlinear, transmedial stories in an effort to find new stories to consume and contribute to. (2) Consumption: The system should facilitate the discourse of nonlinear and transmedial story content, with stories told using multiple media forms, and branching in different directions. (3) Collaborative Creation: 
It should be easy to add new content (text, images, video, etc.) while working with others. (4) Seamless Interfaces: A seamless transition between exploration, consumption, and creation should simplify the process of finding new stories to consume and identifying points in the story to build upon, effectively empowering even traditionally passive content consumers to become prosumers.

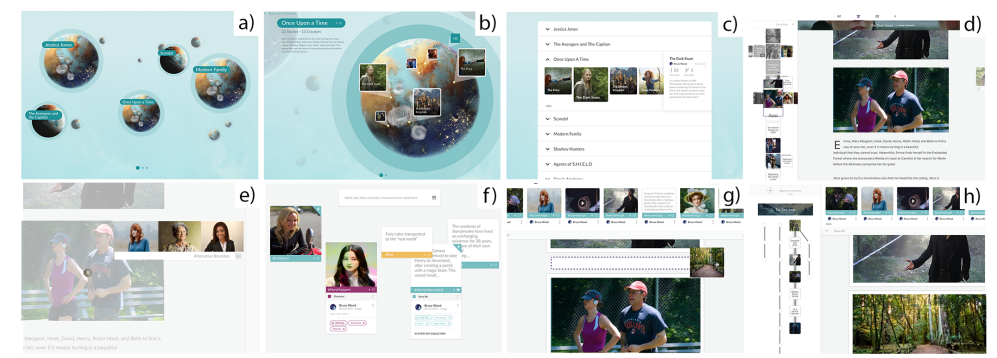

Fig. 1: Visual Interfaces in JUNGLE: (a) A planet-based metaphor for exploring large collections of story worlds. (b) Selecting a story planet allows the user to explore stories (visualized as continents) in that particular story world. (c) A traditional list-style interface for story exploration. (d) Interface for story consumption with left panel visualizing the story graph. (e) User selects an alternate branch of the story to consume. (f) Sketchboard interface for collaborative editing of story bits. (g) Story bits can be easily integrated into existing story. (h) Resulting story. Camera images sourced from Wikimedia under either Creative Commons license or from the Public Domain.

In this paper, we present JUNGLE, an interactive visual platform for the collaborative authoring and consumption of nonlinear, transmedia stories. JUNGLE was iteratively designed to make exploration intuitive, collaboration on ideas straightforward, addition to existing nonlinear and transmedial story content easy, and transitions between exploration and consumption unobtrusive. We introduce a general representation of a story which is visually encoded at different levels of abstraction, each of which caters to a particular interaction task. We explore the benefits and trade-offs of a traditional side-scrolling interface and a planet-based metaphor for exploring stories among multiple story worlds. Stories are consumed using a bi-directional scrolling interface with support for branching. A sketchboard interface is introduced to promote collaboration on individual media elements (story bits), which can then be integrated into a story to fork it into new and exciting directions. In addition, we provide a comprehensive evaluation with two studies: first, a small-scale study to focus on the usability of JUNGLE and next, a large-scale study to demonstrate that long-term engagement in this platform results in (a) collaborative efforts to produce new story worlds, (b) a diverse assortment of linear and branching narratives told through multimedia, and (c) a trend of users transitioning from consumers to prosumers. The primary purpose of JUNGLE and study is to facilitate the collaborative content ideation and creation process. The study scope has been for evaluation of the JUNGLE system as a means of research on enhanced cooperative creation tools, without intent of productization or commercial outcome. The concepts of ownership, access rights, audience adaption, prosumer migration of franchise cannon and wider prosumer media scenarios (e.g. video games) could potentially be developed in the JUNGLE platform, however, these emerging and complex topics are explicitly beyond the scope of this paper. 


\section{Related Work}

Storytelling has been explored from a wide variety of perspectives (both academically and commercially) toward the development of platforms for collaborative usergeneration of interactive stories using different media. Storied Navigation [26] provides an intuitive video editing interface to piece video clips from a text annotated corpus to create compelling video stories. GameBridge [22] presents a nonlinear transmedia story concept within the "Game Of Thrones" story world that combines plot points from the TV show and books. Shwirtz and colleagues [27] explore the impact of social media as a storytelling medium, and its potential for innovative creators to push the boundaries and invent new genres of content and means of connection with audiences. Sadauskas et al. [24] present a prewriting support tool to prepare meaningful writing topics from social media. Balabanovic et al. [6] presents a physical interface for local photo sharing, analogous to a conventional photo album, as well as recording of stories that can be sent to distant friends and relatives. The Graphic StoryWriter (GSW) [30] enables users to create stories through the manipulation of graphic objects in a simulated storybook, relying on a rule-based story engine to guide story development and generate text. CANVAS [16] provides a visual storyboard metaphor for authors to rapidly prototype and visualize 3D animated stories.

Interactive stories [12] strive to transform traditional passive experiences into immersive, engaging experiences where the user can influence the outcome of the narrative. Andrews et al. [5] presents an interactive branching comic for consuming interactive digital narratives. More generally, interactivity is important for exploring new content. Utilizing the Space-Time Continuum creates Adaptive Storyworlds [25] that inspire a framework completely controlled and organized while yet still available to free and open exploration. Mauro and Ardissono [21] developed a co-occurrence graph for the exploration of complex information spaces such as those managed by Geographical Information Systems. Games are also a popular medium of interactive storytelling with extensive research in computational intelligence $[23,15]$ to help authors create compelling freeform interactive narratives. Non-linear choice driven narrative creation systems with procedural generative methods are also emerging for story-centric video games [14]. Lessel et al. [19] investigated how an interacting collaborative audience could influence the course of action in gaming live-streams such as in "Twitch Plays Pokemon".

Some frameworks focus on collaboration. Storeys [11] is a graph-based visualization tool for collaborative story writing that represents stories in a branching tree of individual text sentences. Motif [18] uses storytelling patterns extracted from expert-authored stories to guide novice users. Liu et al. [20] proposed a hypermedia approach to collaborative storytelling activities in social media environments to enable students to integrate the episodes of others to develop different branches of stories into a coherent story. Ensemble, [17] a collaborative writing platform, presents an approach to guiding the diverse perspectives of a creative crowd by using a leader with a high-level vision for a story that articulates constraints. Basaraba proposes a framework to facilitate the collaborative authorship of non-fiction interactive digital narratives [8]. StoryMINE [28] is a platform for creating and consuming multiplayer interactive narrative experiences, in which players experience different narratives. Capturing player interactions in multi-player games [29] brings forth a Multi-Participant Interactive Narrative Framework for games. 
Among commercial platforms, Interlude [1] provides a web-platform for creating interactive video stories. Likewise, Storycanvas [2] facilitates the authoring of interactive stories with a storyboarding tool, and Storyverse [3] hosts professionally created 3D interactive narratives. Existing online platforms and communication systems provide isolated support for collaboration, nonlinearity, or transmedial stories. JUNGLE aims to provide a unified solution for the collaborative creation and consumption of nonlinear transmedia stories that is accessible to everyone.

\section{JUNGLE Platform}

We describe the various capabilities of the JUNGLE platform and the theoretical motivations that influenced them. The careful user studies described in later sections explain the empirical motivations behind this research on data-driven collaboration enhancement.

Story Representation. Stories created, explored, and consumed in JUNGLE are nonlinear and transmedial; each story may be composed of a combination of text, images, audio, video, and other media formats, and may branch in different directions, depending on the viewer's preference. Formally, a story is represented as a directed acyclic graph. This ensures that it is not possible to experience repetitions unless the story is specifically authored in that way. Each node in the story is a story bit: a story atom which corresponds to a single scene in the progression of a story and is composed of text, image, audio, or video. JUNGLE also supports story bits that are an image sequence, or a combination of text and image (e.g., a panel in a comic). Entry and exit nodes indicate the beginning and end of a story, though story graphs can contain multiple entry and exit nodes. Edges represent plausible transitions between story bits. Branching nodes contain multiple outgoing edges to split the story into different directions. A path traversal from any entry node to any exit node represents one plausible linear story. A story world is a collection of stories (multiple, disconnected story graphs) that share a common theme.

Visual Story Metaphors. The general representation of a story described above is visually encoded at different levels of abstraction and presented to the user, catering to particular interaction tasks: exploration, consumption, or collaborative creation. We explore the trade-offs between a traditional scrolling-style exploration interface and a planet-based metaphor for exploring stories among

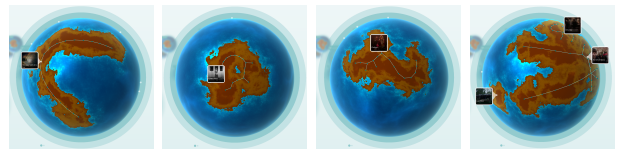

Fig. 2: Planet visualizations of different hypothetical story worlds authored using JUNGLE. (a) A simple linear story for Alice in Wonderland. (b) A branching story in Leila. (c, d) A complex story world comprising many disconnected, nonlinear stories called Once Upon A Time.

multiple story worlds. Stories are consumed by scrolling in a manner similar to current web-based consumption experiences, with the possibility to move horizontally at decision points. A sketchboard (i.e., virtual whiteboard) is proposed for users to collaborate on individual story bits, which can be introduced into the story graph to branch the story into exciting new directions. Engaging users in rich collaborative interactive entertainment environments to facilitate narrative world creation is beginning to develop for video games [4]. In addition to providing an interesting and engaging user experience, for example, our planet visualization serves a few careful purposes in prosumer collaborative 
creation. The use of spherical story graph layout provides a form of focus+context graph visualization (similar to Du et al's iSphere [13]) whilst remaining in 3D with familiar spatial reasoning, and the pictorial terraforming style is aimed at enhancing recall of story forms among collaborators (after observations of Borkin et al [9]).

Story Exploration. Beyond the managed studies of this research we foresee, JUNGLE could contain a plethora of user-generated and professionally curated story worlds, each with a collection of nonlinear, transmedial stories. Navigation and unique visualization systems tailored by task facilitate the exploration of such a large, complex, and heterogeneous dataset. We first provide a traditional flat approach that displays a list of available story worlds. Clicking on a specific story world expands a drop-down menu with title cards for each available story in that world. The user can then browse through the list of title cards by scrolling horizontally, as illustrated in Figure 1(c). We then introduce a planet metaphor for visualizing and exploring stories in JUNGLE. Each story world maps to a planet in the JUNGLE story ecosystem, whose continents refer to disconnected story graphs. Continents are overlaid with graphs to visualize the structures of the corresponding stories. Their shapes are procedurally generated based on their story graphs, but the relative size of a continent is decided based on its rank within the story world (Figure 2). Entry points to these continents are represented by images projected out from the planet and grouped together according to number of story bits shared.

Different visual attributes of the planet metaphor can be mapped to semantics in the underlying stories. For example, story worlds can be ranked in a way that determines their planets' locations between the foreground and the background. Higher ranked planets are pulled towards the foreground, making them appear large than planets in the background (Figure 1(a)). There are several measurements which users may find useful for this work's purpose of enhanced collaborative creation processes, e.g., number of likes, user-creation activity, number of branching story bits, longest path length, and total number of story bits. As the JUNGLE story ecosystem evolves with new content and consumption patterns, the planets dynamically adapt to meet the trends of the users and the continents dynamically reshape themselves to match the structures of the stories. In future, the visualizations could further support the user's preferences, allowing them to control personalized views of their preferred content.

Comparison. Although list-based visualization is pre-established and minimal, there are a couple of limitations that do not suit JUNGLE's usage. First, lists effectively have one monotonic degree of freedom for navigating. A list ordered by measured engagement takes more effort to find stories with lower activity, which in turn may make active stories become increasingly active and therefore potentially isolate other stories. This discourages exploration of new and unknown stories and story worlds. On the other hand, the planet-based visualization does not share this potential limitation. The most popular story (that a list would start at) is the first visible continent on the planet. From this initial view, the user is able to navigate with two and three unrestricted degrees of freedom. Also, although the planet is initially focused on the continent with highest activity measures, the other continents are not arranged by engagement as they would be in a list. This facilitates the unbiased exploration of the story ecosystem.

Another limitation of the list-based visualization is its inability to compare elements. In the planet-based visualization, the relationship between a given continent and every 
other continent can be embedded in its position. For a list to encode the same information, each continent must explicitly store similar continents, leading to redundancy. While the planet metaphor inherently takes more effort to learn than the ubiquitous list representation (in part, due to its novelty), it can potentially encourage more exploration and introduce users to new stories that match preferences under their full control.

Story Consumption Interface The user selects a story to consume (or add to) using one of the two exploration modalities described above. This transitions the user to the consumption interface by default. A unique challenge is to consume stories that contain a combination of media forms, and have multiple branching points. To meet these requirements, the user is presented with two visual representations of the story, as shown in Figure 1(d). The left panel contains a traditional story graph representation which provides a complete perspective of the entire story structure at a glance. The main panel provides a full-size view of each story bit. Scrolling vertically allows the user to proceed down the current story path, consuming story bits (text, images, audio, or videos) in accordance to the progression of the narrative. This is similar to current consumption interfaces which present users with linearly ordered media atoms. In order to support non-linearity in the story structure, the user has the option to scroll horizontally at any decision point in the story graph, where the user may choose to continue along the current story path, or take the story in a new direction. While consuming stories, users may post comments associated with specific story bits for other users to read.

Story Creation. A sketchboard, akin to a virtual whiteboard, is provided for users to work together to create story bits transmedial atoms of a story. The creation of a story bit entails two simple steps. First, the user may either upload media elements or directly type text, and then the user can drag the tile onto the sketchboard.

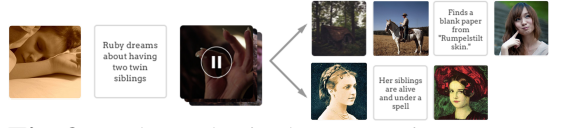

Fig. 3: A hypothetical story using JUNGLE set within a "Once Upon a Time" story world. This simple story contains media composed of text, images, and video, and has two possible outcomes (image source see acknowledgements).

This serves as a visual representation for that particular story bit. Multiple users may simultaneously access the same sketchboard and collaborate in real-time, either through interaction with the story bits, such as adding comments, or by directly communicating with other active users through a traditional chat interface. When a user is editing a story bit in the sketchboard, it is locked, preventing others from simultaneously editing the same story bit. When a user edits a story by adding or removing story bits, a new story branch is created in the story graph, at the junction where the user diverges from the current story. This leaves previously authored stories untouched and mitigates any conflicts and merging issues from arising during story creation. Story bits created in the sketchboard are integrated into a story through a preview version of the consumption interface. Users can simply drag the selected story bit to the corresponding position in the story, thereby creating a new node in the story graph, and possibly introducing a new branching point. Users can preview new content and rapidly iterate through a seamless and collaborative creation-consumption cycle. In cases of alternative views among collaborators, a conflict of ideas can be remedied by branching the story graph (and perhaps rejoining at a future story bit). This particular use captures the potential of branching in stories, allowing for both small-scale changes (e.g., in a single character's 
actions) and large-scale change (e.g., the theme of the narrative). A basic hypothetical story with single branch is shown in Figure 3.

\section{Usability Analysis}

We conducted a small-scale structured user study to evaluate the usability of the different parts of JUNGLE, and the system as a whole. In addition, we performed a comparative analysis of the two interfaces.

Participants. 15 subjects were recruited as part of this study ( 9 male, 6 female) aged between 19 and 34 years $(\mu=$ $24.54, \sigma=5.67)$. Subjects had computer

\begin{tabular}{l} 
Q1 I found it difficult to locate a specific story. \\
Q2 I enjoyed browsing through the story selection. \\
Q3 It was easy to find new content to explore. \\
\hline Q4 It was easy to explore different paths of the same story. \\
Q5 It was easy to consume different types of media. \\
Q6 It was difficult to branch into new continuations of the \\
same story. \\
Q7 It was easy to create stories together with other users. \\
Q8 It was difficult to add different types of media to a story. \\
Q9 It was easy to create stories with different continuations.
\end{tabular}

Table 1: User Study Questions. proficiency and were all novice users with no creative or artistic background.

Experiment Procedure and Task. Each subject was first given a brief introduction to JUNGLE and then asked to perform the following tasks in sequence: (a) Exploration task using Interface A, (b) Exploration task using Interface B, (c) Consumption task, and (d) Creation task. The exploration task required the user to find a specific story within a pre-existing collection of user-generated stories from a previously conducted large-scale study (see description below). The two exploration interfaces (traditional flat interface and planet interface) were randomly ordered for each user to mitigate the effect of learning over successive exploration tasks. For each exploration task, the user was asked to find a different story. Upon selecting a story, the consumption task required the user to use the consumption interface to go through the story while viewing the different transmedial story bits, and exploring the different branching options within the same story. The creation task involved uploading an image to create a new story bit using the sketchboard interface and creating a new branch by integrating them into the story.

After each task, the subject was asked to fill out the System Usability Study (SUS) questionnaire [10], which is a standard measure to quantify the usability of a system. The questionnaire also included a few additional questions (see Table 1) to qualitatively analyze the exploration, consumption, and creation aspects of JUNGLE. Questions were on a 5 point Lik-

\begin{tabular}{l|c|c|c} 
System & $\overline{\mathbf{x}}$ & $\tilde{\mathbf{x}}$ & $\sigma$ \\
\hline Exploration (List) & 77 & 77.5 & 4.93 \\
Exploration (Planets) & 74.83 & 75 & 5.71 \\
Consumption & 73.17 & 75 & 7.29 \\
Creation & 70.67 & 75 & 9.33 \\
JUNGLE & 72.67 & 75 & 10.10 \\
\hline
\end{tabular}

Table 2: Results of SUS Scores ert scale (Strongly Disagree, Disagree, Neutral, Agree, Strongly Agree). Redundant questions were interspersed within the regular set of questions to ensure the responses from the users were valid and not randomly entered. Upon the completion of all 4 tasks and their respective questionnaires, the user completed the SUS form for the whole system. The study was concluded with a short oral debrief, having a total duration of less than 60 minutes per subject.

Table 2 shows the average, median, and standard deviation of the SUS scores for: (a) exploration using traditional flat interface, (b) exploration using planet interface, (c) story consumption, and (d) story creation. While SUS scores are particularly valuable to measure the relative improvement of the system's usability across versions, we can use guidelines [7] (SUS > 70 is considered to be "Good"), coupled with the oral feedback 
from the users at the end of the study, to deduce that the users were able to successfully use all aspects of JUNGLE without hindrance and minimal supervision or training. No significant discrepancy in SUS scores between the specific aspects of JUNGLE was observed, alluding to the relative maturity of the interfaces.

Users were asked additional questions to qualitatively evaluate the various aspects of the system and to compare the two exploration interfaces. The questions are provided in Table 1. Some of the questions expected negative responses, and additional redundant questions were also included in the study for validation purposes. The aggregate statistics of the user's responses are illustrated in Figure 4. According to the study, users find it easy to consume stories in JUNGLE with different media types, and are able to use the interface to explore the different branching points in a nar-

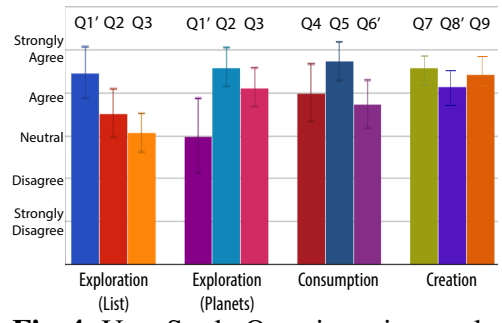

Fig. 4: User Study Questionnaire results. Questions marked with a prime symbol had their response results inverted for consistency $\left(\mathbf{Q}^{\prime}=5\right.$ - Q1). rative. In addition, users find that the sketchboard provides an intuitive interface for collaboration between users and integration of new story bits into the story graph.

List vs. Planet Interface for Story Exploration. We compare the list and planet interface for exploring stories on three factors:

(a) Q1': Ease of finding a specific story,

(b) Q2: Enjoyment in browsing,

\begin{tabular}{|c|c|c|c|c|c|}
\hline \multirow{2}{*}{ 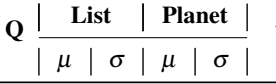 } & & \multirow{2}{*}{ df } & \multirow{2}{*}{ p } & \multicolumn{2}{|c|}{$95 \% \mathrm{CI}$} \\
\hline & & & & LB & UB \\
\hline & & & & & \\
\hline
\end{tabular}

Table 3: Comparative analysis between List and Planet Interface for exploring stories in JUNGLE. The response values of $\mathbf{Q} \mathbf{1}$ have been inverted in this analysis for consistency. results are reported in Table 3, wh shows a significant difference in the mean user scores for all three factors: (a) Q1': $t(25.85)=4.56, p=0.0001$. (b) Q2: $t(26.78)=-3.52, p=0.002$. (c) Q3: $t(21.77)=$ $-3.11, p=0.005$. The results suggest that the users found the traditional list interface easier to use for finding specific stories and the planet interface more engaging for browsing through a large collection of stories or for discovering new, previously unseen story content. This indicates the potential for both interfaces to accommodate different kinds of exploration tasks, as well as creative styles.

\section{User Studies}

In addition to the small-scale study described above, JUNGLE has been rigorously evaluated with 6 studies conducted over a period of 1.5 years, the results of which have been used to iteratively design and improve the platform. We briefly describe the results of the first set of studies, which has led to the current evolution of the JUNGLE platform. 


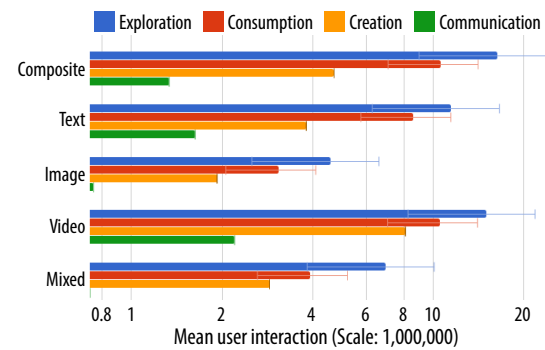

(a) Average user activities for clusters of story planets with unique media signatures.

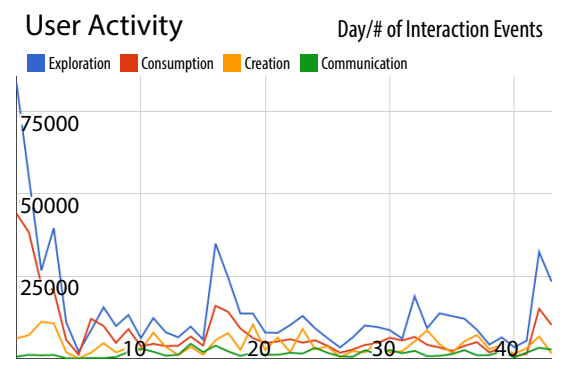

(b) User Activity Trends.

Fig. 5

\subsection{Preliminary Studies}

Five preliminary studies were completed for the purposes of validating the potential of collaborative authoring of nonlinear transmedial stories and to explore group dynamics during the creative process. In the 1st and 2 nd studies, a physical one-day workshop was conducted where users (19 and 18, respectively) were asked to either write a whole story or finish a story. Users were split into groups and asked to work together to create the stories and their conclusions. They were encouraged to produce drawings, recordings, and take any material they liked from free resources on the Internet. Surveys from these workshop studies always elicited positive feedback towards the collaborative environment and the task. In the 3rd and 4th studies, individual user tests were conducted where users ( 5 and 6 , respectively) of different nationalities were asked to interact with a preliminary version of the planet-based story exploration interface. Following a short tutorial, users found the planet metaphor intuitive and found the notion of branching stories intriguing. In the 5th study, a preliminary version of JUNGLE with exploration, consumption, and creation capabilities was released to 190 users from all over the world. Users were invited to browse through the story worlds and continue developing the stories in any way that they wished using images, text, audio, or video. Collaboration was not supported in this version, and users could not interact with each other. 80 out of 190 users developed stories, while all users explored and consumed content in JUNGLE.

\subsection{Large-scale User Study}

The sixth study was conducted with 354 users, who were given free access to the JUNGLE platform over a period of six weeks. Users ranged from 16 to 71 years old $(\mu=32, \sigma=8.54) .62 \%$ of the users were female, $37.7 \%$ male, and $0.3 \%$ identified as gender queer. They were asked to test the platform with no further instructions.

User engagement. Users spent an average of 71 minutes on the platform with the top 30 users spending an average of 380 minutes on the platform. Users visited 11498 scenes and contributed 1228 comments, 243 likes, and 1021 story path ratings.

Media. There were 50 user-created story planets containing at least 1 story (the other planets were disregarded in this analysis). These planets consisted of a rich diversity of media with 439 text bits, 1033 images, 38 videos, 214 composite story bits (a combination of image and text), and 43 sequence story bits (a slide show of images). The media 


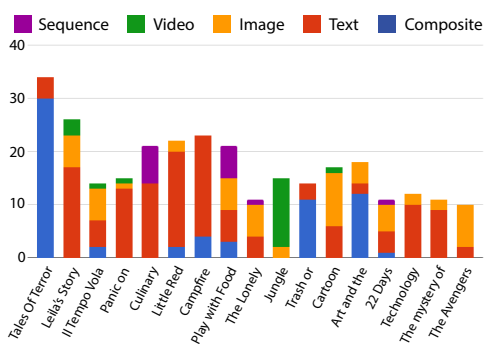

(a) Media signatures of some user-authored stories.

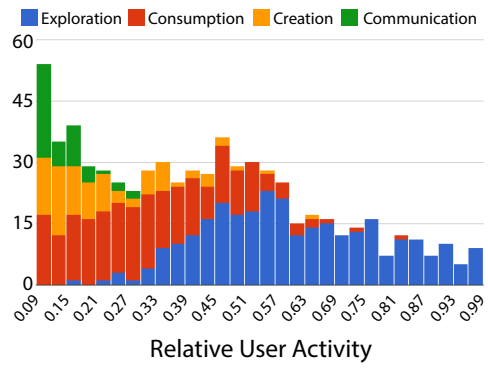

(b) Histogram of relative user activity.

Fig. 6

signatures (i.e., relative distributions of media elements) for these planets are illustrated in Figure 6a. We cluster the 50 story worlds together into the following categories: (a) Text-centric (20), (b) Image-centric (6), (c) Video-centric (2), (d) Composite-centric (8), and (e) Mixed (14). Figure 5a provides the mean user activities for the different categories of story planets. Multiple trends stand out from the various activities of users across the planets in our research study's findings. Exploration dominates the activities of users across all planet archetypes, perhaps owing to the relative novelty of the JUNGLE research prototype. Video-centric stories were measured among study participants to have the largest consumption times, on average, but also elicit maximum consumption from participants. Video-centric planets also recorded the maximum communication between users. Figure 7 illustrates stories using different combinations of media.

Story Structure. We analyze the structure of the story graphs that were created by the users in terms of number of story nodes $N$, connectivity $N_{c}$ (measured in terms of number of subgraphs where any two nodes in a subgraph were connected to each other through a path traversal), and maximum degree $d_{\max }$ of a story node. The relevant statistics are reported in Table 4. The largest story, "Tales of Terror" had 38 nodes (made up of text and composite story bits). Stories had a calculated average maximum degree of 2.38, with the majority of stories having between 2 and 3 story paths, and the maximum degree observed 6 story paths. We observe that a majority of the stories had some degree of nonlinearity $\left(d_{\max }>1\right)$, with nearly

\begin{tabular}{l|c|c|c} 
Metric & $N$ & $N_{c}$ & $d_{\max }$ \\
\hline Max & 38 & 6 & 6 \\
Min & 2 & 1 & 1 \\
Mean & 9.78 & 2.06 & 2.38 \\
Median & 7.5 & 2 & 2 \\
\hline
\end{tabular}

Table 4: Aggregate story graph metrics across all user-authored story planets. $N$ : number of nodes in a story graph. $N_{c}$ : number of connected subgraphs in a story graph.$d_{\max }$ : Maximum degree of a story node.

10 of the 50 planets having more than 8 decision points at various stages in the story. Figures 7 and 9 illustrate the graph structure of some stories that were created by users in JUNGLE. The structure of stories ranged from strictly linear experiences to complex branching stories with multiple decision points. More complex graph-theoretic measures may be used to glean additional insights into the structure of the stories created among users, which is beyond the scope of this study.

User Activity. Within the scope of this study with careful attention to privacy, we temporarily logged the activity profiles of users according to activity type (exploration, consumption, creation, or communication) that the users were currently engaged in. The activity trends over the duration of the study are illustrated in Figure 5b. Following an 


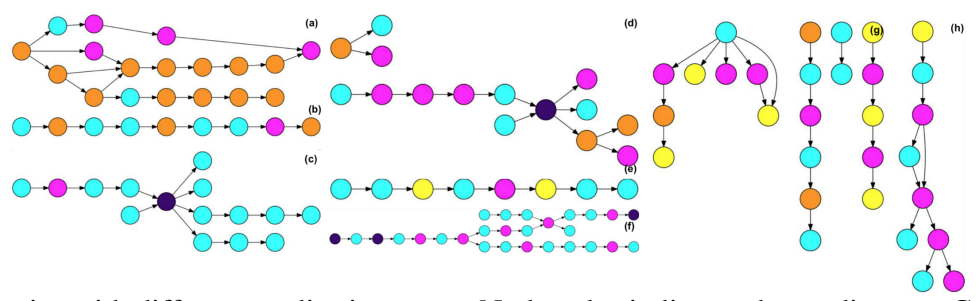

Fig. 7: Stories with different media signatures. Node color indicates the media type: Composite (orange), Text (cyan), Image (magenta), Video (indigo), and Sequence (yellow). The story titles are as follows: (a) Art and the Unconscious, (b) Mystery of the Lost Wallet, (c) Panic on Planet Parmeson, (d) Tempo Vola, (e) A Tale of Two Lovers, (f) Leila's Story, (g) Play with Food.

initial surge in user activity, we see a steady pattern of exploration, consumption, and creation across users, with periodic spikes that might be attributed to new story initiatives by active users. Note that no professional content was added by us for the duration of this study. All content was user-generated for this hypothetical collaborative creation study only, without any intent of formal production. A histogram of the relative user activity for all users is provided in Figure 6b. While a large percentage of the users spend the majority of their time exploring and consuming content, a significant number of active users communicate and collaborate with one another to create new story content.

User Up-Conversion. We measure the creation to consumption ratio of all users over the study duration, and observe its trend (Figure 8). As demonstrated by the results, users show a marked increase in creating new story content with time spent on the platform. This is indicative of user up-conversion where traditionally passive users who are more likely to consume content, begin to actively create new story content, together with other users. This may in part be due to the seam-

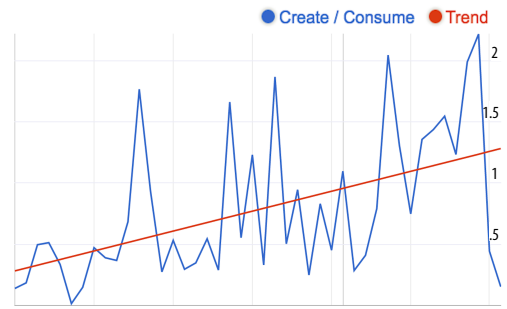

Fig. 8: Creation to consumption trend in user activity for the duration of study. less coupling of the exploration, consumption, and creation interaction tasks in the platform. JUNGLE offers the agency for users, who prefer to consume content, to actively engage with other users and create new stories.

Collaboration. JUNGLE allows users to work together on the same story by contributing new story bits and commenting on existing stories, which influences how the stories evolve over time. We use a simple measure of collaboration as the number of users who contributed story bits to a story. The number of users that worked together to create each story planet during this study ranged from 1 to 6 collaborators $(\mu=1.476, \sigma=$ 1.145). 461 users worked on 65 planets while there were 49 planets with only 1 author. Figure 9 illustrates a set of story graphs, created by multiple users, where the node color denotes a specific user. Different creation patterns are observed where users may work independently on different branches of the same story graph, or collaborate to iteratively contribute to the progression of the same story path. There are many factors that are not considered in this study. For instance, multiple users can contribute to the same story bit using the sketchboard interface. Additionally, an indirect form of collaboration occurs where users may not explicitly add new content, but influence the evolution of the story by commenting on existing stories. 


\section{Conclusion}

JUNGLE is an interactive visual platform that allows both novice users as well as creative professionals to collaboratively create and consume branching story structures that take the form of various combinations of video, images, and text. JUNGLE has been extensively evaluated in both controlled studies and large-scale free-form experiments with hundreds of users over several weeks of activity. Our analysis shows that JUNGLE delivers users the ability to create and consume nonlinear, transmedial stories. In addition, we observe a marked increase in the creation activities of users with time spent on the platform (the longest study lasted 45 days), which is indicative of user up-conversion.

While the platform is now in a mature state that has been tested and refined across studies spanning 18 months, we will continue to improve JUNGLE based on user feedback. For example, story ownership and access rights will be integrated and studied for more perspective on the collaborative creation process. Our latest results reveal that users prefer different interfaces (a list-style interface vs. a planet metaphor)

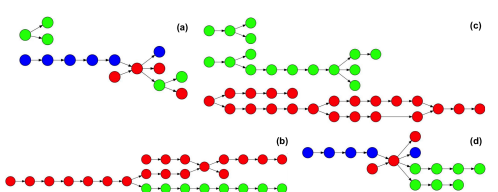

Fig. 9: Different story graphs that were authored by JUNGLE users. Node colors indicate the user who was responsible for creating the story bit. (a) Tempo Vola, (b) Leila's Story, (c) Tales of Terror, (d) Panic on Planet Parmeson. depending on the exploration task. For future versions of the platform, we would like to consolidate both these interfaces for exploring stories into a single, unified experience.

JUNGLE opens up several exciting avenues of future research in story telling and the analysis of the creation and consumption activities of different user groups. We are interested in studying how new stories can be recommended to users (both for creation and consumption), based on their past activities. Our story representation makes it possible for existing stories to adapt dynamically and be personalized based on the viewers preferences. In future, analysing whether users from different age groups have similar or different preferences in interaction UI, content creation, and consumption behaviors. Further analysis into the media signatures of stories authored will provide insights into new genres and story archetypes of JUNGLE.

\section{Acknowledgements}

Kapadia has been funded in part by NSF IIS-1703883 and NSF S\&AS-1723869. Many additional colleagues served in the development of this study, including Max Grosse, Rebekkah Laeuchi, David Sinclair, Isa Simo, Mark Miller, Soheyon Jeong, Adriano Galati, Maria Cabral, Barbara Solenthaler, Miquel Farre, Samir Mahmalat, Roberto Sousa, Nam Wook Kim, Tanja Kaser, Ancona Marco, Alesia Marra, Maggie Kosek, Maurizio Nitti, Klinger Severin, Anslem Grundhofer, Peter Kaufmann, Hanspeter Pfister, and Bob Sumner. We thank Wikimedia with images sourced as either public domain or under creative common license with attribution. Photographers include Magnus Manske, Christian Madison, Ed Yourdon, Sardaka, Puram Yun, and Pepe Robles. 


\section{References}

1. Interlude. https://interlude. fm (2016)

2. Storycanvas. http://thetoolkitproject.com/tool/story-canvas (2016)

3. Storyverse. http://www. storyversestudios.com/ (2016)

4. Acharya, D., Wardrip-Fruin, N.: Building worlds together: Understanding collaborative co-creation of game worlds. In: Proceedings of the 14th International Conference on the Foundations of Digital Games. pp. 45:1-45:5. FDG '19, ACM, New York, NY, USA (2019). https://doi.org/10.1145/3337722.3337748, http: //doi . acm . org/10.1145/ 3337722.3337748

5. Andrews, D., Baber, C.: Visualizing interactive narratives: Employing a branching comic to tell a story and show its readings. In: Proceedings of the $32 \mathrm{Nd}$ Annual ACM Conference on Human Factors in Computing Systems. pp. 1895-1904. CHI '14, ACM, New York, NY, USA (2014). https://doi.org/10.1145/2556288.2557296, http: //doi . acm . org/10. 1145/ 2556288.2557296

6. Balabanović, M., Chu, L.L., Wolff, G.J.: Storytelling with digital photographs. In: Proceedings of the SIGCHI Conference on Human Factors in Computing Systems. pp. 564-571. CHI '00, ACM, New York, NY, USA (2000). https://doi.org/10.1145/332040.332505, http://doi . acm.org/10.1145/332040.332505

7. Bangor, A., Kortum, P., Miller, J.: Determining what individual sus scores mean: Adding an adjective rating scale. J. Usability Studies 4(3), 114-123 (May 2009), http : / /dl . acm . org/ citation. cfm?id=2835587.2835589

8. Basaraba, N.: A Framework for Creative Teams of Non-fiction Interactive Digital Narratives: 11th International Conference on Interactive Digital Storytelling, ICIDS 2018, Dublin, Ireland, December 5âĂŞ8, 2018, Proceedings, pp. 143-148 (01 2018). https://doi.org/10.1007/978-3030-04028-4 1

9. Borkin, M.A., Vo, A.A., Bylinskii, Z., Isola, P., Sunkavalli, S., Oliva, A., Pfister, H.: What makes a visualization memorable? IEEE Transactions on Visualization and Computer Graphics 19(12), 2306-2315 (2013)

10. Brooke, J.: Sus: A quick and dirty usability scale (1996)

11. Cheng, J., Kang, L., Cosley, D.: Storeys: Designing collaborative storytelling interfaces. In: CHI '13 Extended Abstracts on Human Factors in Computing Systems. pp. 3031-3034. CHI EA '13, ACM, New York, NY, USA (2013). https://doi.org/10.1145/2468356.2479603, http://doi.acm.org/10.1145/2468356.2479603

12. Clanton, C., Marks, H., Murray, J., Flanagan, M., Arble, F.: Interactive narrative: Stepping into our own stories. In: CHI 98 Conference Summary on Human Factors in Computing Systems. pp. 88-89. CHI '98, ACM, New York, NY, USA (1998). https://doi.org/10.1145/286498.286543, http://doi . acm.org/10.1145/286498. 286543

13. Du, F., Cao, N., Lin, Y.R., Xu, P., Tong, H.: isphere: Focus+context sphere visualization for interactive large graph exploration. In: Proceedings of the $2017 \mathrm{CHI}$ Conference on Human Factors in Computing Systems. pp. 2916-2927. CHI '17, ACM, New York, NY, USA (2017). https://doi.org/10.1145/3025453.3025628, http: //doi . acm . org/10.1145/ 3025453.3025628

14. Garbe, J., Kreminski, M., Samuel, B., Wardrip-Fruin, N., Mateas, M.: Storyassembler: An engine for generating dynamic choice-driven narratives. In: Proceedings of the 14th International Conference on the Foundations of Digital Games. pp. 24:1-24:10. FDG '19, ACM, New York, NY, USA (2019). https://doi.org/10.1145/3337722.3337732, http: //doi.acm.org/10.1145/3337722.3337732 
15. Kapadia, M., Falk, J., Zünd, F., Marti, M., Sumner, R.W., Gross, M.: Computerassisted authoring of interactive narratives. In: Proceedings of the 19th Symposium on Interactive 3D Graphics and Games. pp. 85-92. i3D '15, ACM, New York, NY, USA (2015). https://doi.org/10.1145/2699276.2699279, http://doi . acm . org/10.1145/ 2699276.2699279

16. Kapadia, M., Frey, S., Shoulson, A., Sumner, R.W., Gross, M.: CANVAS: Computer-Assisted Narrative Animation Synthesis. In: ACM SIGGRAPH/Eurographics Symposium on Computer Animation. SCA '16, Eurographics (2016)

17. Kim, J., Cheng, J., Bernstein, M.S.: Ensemble: Exploring complementary strengths of leaders and crowds in creative collaboration. In: Proceedings of the 17th ACM Conference on Computer Supported Cooperative Work \&\#38; Social Computing. pp. 745-755. CSCW '14, ACM, New York, NY, USA (2014). https://doi.org/10.1145/2531602.2531638, http: //doi.acm.org/10.1145/2531602.2531638

18. Kim, J., Dontcheva, M., Li, W., Bernstein, M.S., Steinsapir, D.: Motif: Supporting novice creativity through expert patterns. In: Proceedings of the 33rd Annual ACM Conference on Human Factors in Computing Systems. pp. 1211-1220. CHI '15, ACM, New York, NY, USA (2015). https://doi.org/10.1145/2702123.2702507, http: //doi . acm . org/10. 1145/ 2702123.2702507

19. Lessel, P., Mauderer, M., Wolff, C., Krüger, A.: Let's play my way: Investigating audience influence in user-generated gaming live-streams. In: Proceedings of the 2017 ACM International Conference on Interactive Experiences for TV and Online Video. pp. 51-63. TVX '17, ACM, New York, NY, USA (2017). https://doi.org/10.1145/3077548.3077556, http://doi.acm.org/10.1145/3077548.3077556

20. Liu, C.C., Liu, K.P., Chen, W.H., Lin, C.P., Chen, G.D.: Collaborative storytelling experiences in social media: Influence of peer-assistance mechanisms. vol. 57, pp. $1544-$ 1556 (2011). https://doi.org/https://doi.org/10.1016/j.compedu.2011.02.002, http: //www . sciencedirect.com/science/article/pii/S0360131511000406

21. Mauro, N., Ardissono, L.: Session-based suggestion of topics for geographic exploratory search. In: 23rd International Conference on Intelligent User Interfaces. pp. 341-352. IUI '18, ACM, New York, NY, USA (2018). https://doi.org/10.1145/3172944.3172957, http: //doi.acm.org/10.1145/3172944.3172957

22. Miles, R., Cason, A., Chan, L., Li, J., McDonnell, R., Murray, J., Wang, Z.: Gamebridge: Converging toward a transmedia storytelling experience through gameplay. In: Proceedings of the ACM International Conference on Interactive Experiences for TV and Online Video. pp. 105111. TVX '16, ACM, New York, NY, USA (2016). https://doi.org/10.1145/2932206.2932209, http://doi.acm.org/10.1145/2932206.2932209

23. Riedl, M.O., Young, R.M.: From Linear Story Generation to Branching Story Graphs. IEEE Computer Graphics and Applications 26(3), 23-31 (Jun 2006)

24. Sadauskas, J., Byrne, D., Atkinson, R.K.: Mining memories: Designing a platform to support social media based writing. In: Proceedings of the 33rd Annual ACM Conference on Human Factors in Computing Systems. pp. 3691-3700. CHI '15, ACM, New York, NY, USA (2015). https://doi.org/10.1145/2702123.2702383, http://doi . acm . org/10.1145/ 2702123.2702383

25. Schoenau-Fog, H.: Adaptive storyworlds. In: Schoenau-Fog, H., Bruni, L.E., Louchart, S., Baceviciute, S. (eds.) Interactive Storytelling. pp. 58-65. Springer International Publishing, Cham (2015)

26. Shen, E.Y.T., Lieberman, H., Davenport, G.: What's next?: Emergent storytelling from video collection. In: Proceedings of the SIGCHI Conference on Human Factors in Computing Systems. pp. 809-818. CHI '09, ACM, New York, NY, USA (2009). https://doi.org/10.1145/1518701.1518825, http://doi . acm. org/10.1145/ 1518701.1518825 
27. Shwirtz, J.: Empowering storytellers with social media. In: Proceedings of the ACM International Conference on Interactive Experiences for TV and Online Video. pp. 1-1. TVX '15, ACM, New York, NY, USA (2015). https://doi.org/10.1145/2745197.2749467, http://doi.acm.org/10.1145/2745197.2749467

28. Spawforth, C., Gibbins, N., Millard, D.: Storymine: A system for multiplayer interactive narrative experiences (12 2018)

29. Spawforth, C., Millard, D.E.: A framework for multi-participant narratives based on multiplayer game interactions. In: Nunes, N., Oakley, I., Nisi, V. (eds.) Interactive Storytelling. pp. 150-162. Springer International Publishing, Cham (2017)

30. Steiner, K.E., Moher, T.G.: Graphic storywriter: An interactive environment for emergent storytelling. In: Proceedings of the SIGCHI Conference on Human Factors in Computing Systems. pp. 357-364. CHI '92, ACM, New York, NY, USA (1992). https://doi.org/10.1145/142750.142831, http://doi . acm.org/10.1145/142750. 142831 